\title{
PRÁTICAS OR CAMENTÁRIAS APLICADAS EM EMPRESAS HOTELEIRAS NO BRASIL: UM ESTUDO NA CIDADE DE BALNEÁRIO CAMBORIÚ - SC
}

\author{
BUDGET PRACTICES APPLIED TO HOTEL COMPANIES IN BRAZIL: A STUDY IN THE TOWN OF \\ BALNEÁRIO CAMBORIÚ \\ LAS PRÁCTICAS PRESUPUESTARIAS APLICADAS EN EMPRESAS HOTELERAS EN BRASIL: UN \\ ESTUDIO EN LA CIUDAD DE BALNEÁRIO CAMBORIÚ
}

Maurício Mello Codesso

Mestrando no Programa de Pós-Graduação em Contabilidade - UFSC

E-mail: mmcodesso@gmail.com

Rogério João Lunkes

Professor no Programa de Pós-Graduação em Contabilidade - UFSC Pós-Doutor na Universidade de Valência (2011)

E-mail: rogeriolunkes@hotmail.com

Ricardo Suave

Mestrando no Programa de Pós-Graduação em Contabilidade - UFSC

E-mail: ricardosuave@outlook.com

\section{RESUMO}

O setor turístico representa importante papel na economia brasileira e, especialmente no Estado de Santa Catarina, a cidade de Balneário Camboriú recebe centenas de milhares de turistas estrangeiros todos os anos. Como os hotéis exercem papel de destaque nessa atividade, o presente estudo tem como objetivo fornecer evidências sobre as práticas orçamentárias em empresas hoteleiras na cidade de Balneário Camboriú (SC). Para atingir este objetivo, uma pesquisa empírica foi realizada, com o envio de questionário a todos os hotéis associados à Associação da Indústria Hoteleira de Santa Catarina (ABIH-SC), sendo a população inicial formada por 17 hotéis de Balneário Camboriú. A partir desta população inicial, foram selecionados 11 hotéis que possuem orçamento formal, com o retorno de 6 questionários. A estatística descritiva foi aplicada sobre os resultados, visando melhorar a base das discussões. Os resultados mostram que a maioria dos hotéis possui um processo formal de orçamento, tendo o bottom-up como processo orçamentário predominante. A pesquisa revela ainda quais as bases para a elaboração do orçamento e dos aspectos relacionados às etapas de execução e controle, bem como suas finalidades.

PALAVRAS-CHAVE: Orçamento. Planejamento. Hotéis.

\section{ABSTRACT}

The tourism sector represents an important role in the Brazilian economy, and this is particularly true of the state of Santa Catarina, and more specifically, the town of Balneário Camboriú, which receives hundreds of thousands of foreign tourists every year. Given that hotels play a prominent role in this activity, this present study aims to provide evidence of practices in budget at hotel companies in the town. This was done through an empirical study, in which a survey was sent out to all the member hotels of the ABIH-SC (Hotel Industry Association of Santa Catarina). The initial study population consisted of 17 hotels in Balneário Camboriú, from which 11 hotels were selected that have a formal budget. Of these, 6 
questionnaires were returned. Descriptive statistics were applied to the results, to improve the basis for discussions. The results show that most hotels have a formal budget, the bottom-up style of budgeting being predominant. The research also reveals the bases used when establishing the budget, and aspects related to the stages of execution and control, as well as their purposes.

KEYWORDS: Budgeting. Planning. Hotels.

\section{RESUMEN}

El sector turístico desempeña un importante papel en la economía brasileña, y especialmente en el Estado de Santa Catarina, donde la ciudad de Balneário Camboriú recibe a cientos de miles de turistas extranjeros todos los años. Como los hoteles ejercen un papel de destaque en esa actividad, el presente estudio tiene como objetivo proporcionar evidencias acerca de las prácticas presupuestarias en empresas hoteleras de la ciudad de Balneário Camboriú (SC). Para alcanzar este objetivo se realizó una investigación empírica con el envío de un cuestionario a todos los hoteles asociados a la Asociación de la Industria Hotelera de Santa Catarina (ABIH-SC), cuya población inicial estaba formada por 17 hoteles de Balneário Camboriú. A partir de esta población inicial, fueron seleccionados 11 hoteles que poseen presupuesto formal, con el retorno de 6 cuestionarios. La estadística descriptiva fue aplicada sobre los resultados con el propósito de mejorar la base de las discusiones. Los resultados muestran que la mayoría de los hoteles posee un proceso formal de presupuesto, con el bottom-up como proceso presupuestario predominante. La investigación revela también cuáles son las bases para la elaboración del presupuesto y de los aspectos relacionados a las etapas de ejecución y control, así como sus finalidades.

PALABRAS CLAVE: Presupuesto. Planeamiento. Hoteles.

\section{INTRODUÇÃO}

O orçamento é um dos sistemas de planejamento e controle mais antigo adotado pelas empresas (OTLEY, 1994; DAVILA; FOSTER, 2005 e 2007; SANDINO, 2007; KING; CLARKSON; WALLACE, 2010). Além disto, continua sendo um dos instrumentos fundamentais e amplamente utilizado pelas organizações na gestão das operações (SZYCHTA, 2002; ABDEL-KADER; LUTHER, 2006; UYAR, 2009; SIVABALAN et al., 2009; LIBBY; LINDSAY, 2010; OSTERGREN; STENSAKER, 2011; UYAR; BILGIN, 2011). O sistema orçamentário é um dos mecanismos mais utilizados pelos gestores para controlar os custos e melhorar o desempenho (KING; CLARKSON; WALLACE, 2010).

O orçamento é um importante instrumento de planejamento, execução e controle, com a função de converter os objetivos estratégicos em metas e valores operacionais, gerando um guia para as operações e as decisões dos gestores. Horngren, Foster e Datar (2000) definem orçamento como sendo a expressão quantitativa de um plano de ação futuro da administração para determinado período.

Embora seja um instrumento amplamente utilizado pelas organizações, também é frequentemente alvo de críticas. Há estudos de mostram que os problemas do orçamento estão relacionados à forma em que são utilizados (HORNGREN; FOSTER; DATAR, 2000), enquanto outros vão além e relatam que os processos orçamentários apresentam falhas (HOPE; FRASER, 2003; HANSEN; OTLEY; VAN Der STEDE, 2003). Para Jones (2008a), estas abordagens apresentam soluções diferentes, mas também compartilham muitas das preocupações do orçamento empresarial. Além disto, apesar deste debate, o orçamento empresarial continua ainda a ser amplamente utilizado nas organizações (JONES, 2008b; UYAR; BILGIN, 2011; CASTRO et al., 2012). Para Libby e Lindsay (2010), embora o orçamento possa apresentar problemas, as organizações preferem adaptar a sua utilização para dar conta desses problemas, em vez de abandoná-lo completamente.

Alguns processos orçamentários tradicionais, como o empresarial, são considerados incapazes de atender às demandas do ambiente competitivo e de rápidas mudanças e são criticados por impedir 
alocação eficiente de recursos e incentivar um comportamento disfuncional, com decisões míopes, além de permitir os jogos orçamentários (gamesmanship). No entanto o orçamento ainda é considerado um imperativo organizacional e há pouca evidência empírica de que as organizações vão abandoná-lo no futuro (De WAAL; HERMKENS-JANSSEN; VAN De VEN, 2011; KORNACKER et al., 2011).

Em geral, nestes debates há a percepção que as organizações precisam melhorar seus processos orçamentários, visando torná-lo mais eficaz e eficiente, e com isto obter mais benefícios (UYAR; BILGIN, 2011). Este ganho em eficiência pode ser obtido com mudanças nas formas e nas práticas aplicadas no planejamento, na execução e no controle do orçamento. Estudos realizados por Umapathy (1987) e King, Clarkson e Wallace (2010) provaram que as empresas de sucesso, de fato, adotavam algumas práticas que faziam com que seus sistemas orçamentários fossem mais eficazes. Entre as práticas destacadas, estão a geração de compromisso, o alinhamento entre os níveis da organização, a adoção de procedimentos detalhados e abrangentes na elaboração, além da análise das variações e da tomada de ações corretivas.

Embora as práticas de orçamento sejam estudadas na literatura (SCHMIDGALL et al.,1996; SCHMIDGALL; DeFRANCO, 1998; JONES, 1998; JONES, 2008; SUBRAMANIAM et al., 2002; WINATA; MIA, 2005; YUEN, 2006; PAVLATOS; PAGGIOS, 2009; STEED; GU, 2009; UYAR; BILGIN, 2011; e CASTRO et al., 2012), precisam ser pesquisados de forma mais ampla, visando identificar e discutir práticas e processos mais eficientes em diferentes setores da economia.

Um setor que passou a ser destaque em diversas regiões do Brasil é o turismo. Esse setor, e em particular com a contribuição das empresas hoteleiras, trata-se de um setor importante para a economia de muitos países. No Brasil, o turismo representa aproximadamente $5 \%$ do Produto Interno Bruto (PIB). Já a região de Balneário Camboriú, onde foi realizado o estudo, é um destino importante, visitado por mais de 1 milhão de turistas estrangeiros por ano, constituindo $20 \%$ do número total de turistas recebidos pelo Brasil. Santa Catarina é o segundo Estado que mais recebe turistas estrangeiros no Brasil (EMBRATUR, 2011).

Dessa forma, o presente trabalho possui como objetivo explorar as práticas orçamentárias em empresas hoteleiras na cidade de Balneário Camboriú (SC). Justifica-se o trabalho, pois visa contribuir para a compreensão das práticas de orçamento em empresas hoteleiras no Brasil, que têm recebido relativamente pouca atenção na literatura se comparado ao uso de práticas mais sofisticadas em outros países. Ao comparar os resultados com a literatura e outros trabalhos empíricos anteriores, poderá permitir explorar aspectos ainda não incorporados pelas empresas hoteleiras brasileiras. Em termos de implicações práticas, o estudo busca auxiliar os gestores das empresas na escolha de métodos adequados, visando tomar a melhor decisão de investimento.

A avaliação pode mostrar lacunas teoria-prática no detalhamento dos métodos e técnicas de orçamento. Além disso, pode ser valioso para fazer um balanço sobre a incorporação de novos conceitos que têm sido desenvolvidos ao longo dos últimos anos, como o orçamento de base zero. Assim, este trabalho pode oferecer um diagnóstico da realidade atual, o que, com a síntese e a integração de outros estudos sobre o tema, possibilitará novas comparações e oportunidades de pesquisa e aplicações práticas, visando melhorar o planejamento em empresas hoteleiras brasileiras.

Além disso, conforme enfatizado por Araújo (2001), ao combinar este planejamento na redução de custos, empresas hoteleiras que o adotam se tornam diferentes de sua clientela, oferendo serviços de maior qualidade. Em sua estrutura, o trabalho apresenta, além dessa introdução, na segunda seção o referencial teórico; a terceira consiste na exposição dos procedimentos metodológicos utilizados pela pesquisa. Na quarta seção apresentam-se os resultados da pesquisa e na quinta são feitas as considerações finais.

\section{REVISÃO DA LITERATURA}

O orçamento envolve um processo de planejamento, execução e controle das operações. Horngren, Foster e Datar (2000) definem orçamento como sendo a expressão quantitativa de um plano de ação futuro da administração para determinado período. A orçamentação também pode ser um meio de converter o plano estratégico da empresa em padrões mensuráveis (ADAMS, 2006). 
O orçamento representa o esforço da organização para quantificar a captação e a utilização dos recursos, que aborda dois fins de gestão, planejamento e controle (GARRISON et al., 2008). É uma previsão do período futuro de um ano, que inclui marketing e outras informações que levam ao estabelecimento de objetivos e à medição de desempenho (HARRIS, 1999). Como parte integrante do orçamento, a previsão é necessária para o planejamento das formas mais eficazes e eficientes para atender o volume de vendas (SCHMIDGALL, 2006).

Os estudos sobre práticas de orçamento em empresas hoteleiras têm sido realizados em diversos países, como nos Estados Unidos (SCHMIDGALL; DeFRANCO, 1998; STEED; GU, 2009), Estados Unidos e Escandinávia (SCHMIDGALL et al. 1996), Reino Unido e Estados Unidos (Jones, 1998; JONES, 2008), na Austrália (SUBRAMANIAM et al., 2002; WINATA; MIA, 2005); China (YUEN, 2006), Grécia (PAVLATOS; PAGGIOS, 2009), Turquia (UYAR; BILGIN, 2011) e Brasil (CASTRO et al., 2012). A Tabela 1 mostra as principais práticas de elaboração do orçamento utilizadas pelas empresas hoteleiras em diferentes partes do mundo.

Quanto à execução do orçamento, pode-se destacar os estudos de Jones (2008b), Uyar e Bilgin (2011) e Castro et al. (2012), que diagnosticaram práticas relacionadas à avaliação do processo de execução, objetivos e finalidades, informações utilizadas e aspectos comportamentais. Já em relação à avaliação de desempenho: análises sobre a utilização de bechmarking, papel desempenhado pelo orçamento no monitoramento, principais medidas de desempenho utilizadas, responsáveis pela análise das variações.

Pode-se ainda destacar três outros trabalhos sobre orçamento. O primeiro foi um estudo realizado por Subramaniam et al. (2002), com 91 gestores de empresas hoteleiras da Austrália sobre o papel e o impacto da estrutura descentralizada, necessidade de atingir metas e orçamento participativo sobre o comportamento organizacional. Os resultados mostraram relações diretas e positivas das duas variáveis, estrutura descentralizada, necessidade de atingir metas para a realização e participação no orçamento. Além disto, o orçamento participativo pode ter uma relação direta e positiva com o comportamento organizacional.

Quadro 1 - Práticas empresariais de orçamento

\begin{tabular}{|c|c|c|c|c|c|c|c|c|c|c|c|}
\hline \multicolumn{2}{|c|}{$\begin{array}{l}\text { PRINCIPAIS QUESTÕES PESQUISADAS } \\
\text { SOBRE ELABORAÇÃO DO ORÇAMENTO }\end{array}$} & 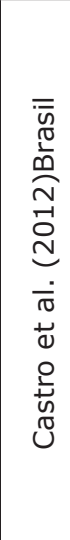 & 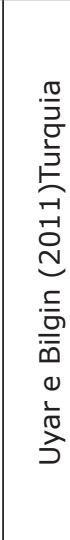 & 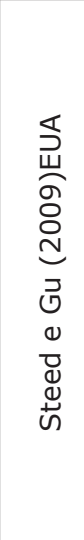 & 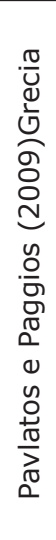 & 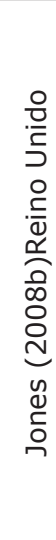 & 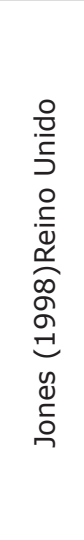 & 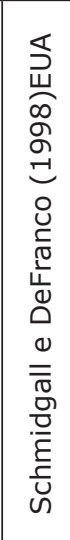 & 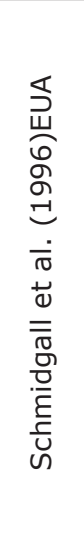 & 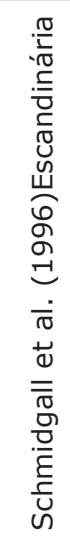 & 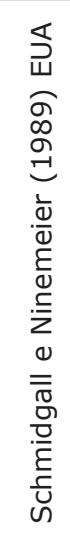 \\
\hline \multicolumn{2}{|c|}{ Manual de orçamento } & 100 & 63 & & & & & & & & 50 \\
\hline \multicolumn{2}{|c|}{ Comissão de orçamento } & 79 & 87 & & & & & & & & \\
\hline \multirow{4}{*}{$\begin{array}{l}\text { Tempo de } \\
\text { preparação do } \\
\text { orçamento }\end{array}$} & Menos de 40 horas & 69 & & 15,0 & & & & & & & \\
\hline & 40 e 80 horas & 23 & & 27,5 & & & & & & & \\
\hline & 81 a 120 horas & 0 & & 27,5 & & & & & & & \\
\hline & Mais de 120 horas & 8 & 80 & 30,0 & & & & & & & \\
\hline \multirow{3}{*}{$\begin{array}{l}\text { Formas de } \\
\text { elaborar o } \\
\text { processo orça- } \\
\text { mentário }\end{array}$} & Top-down & 29 & 24 & 2.4 & & 26 & 13.6 & 4.5 & 4.5 & 4 & 13.3 \\
\hline & Bottom-up & 57 & 34 & 16.7 & & 36 & 54.6 & 64 & 79.5 & 64 & 56.7 \\
\hline & Combinação & 14 & 42 & 78.6 & & 39 & 31.8 & 28 & 16 & 28 & 26.7 \\
\hline
\end{tabular}




\begin{tabular}{|c|c|c|c|c|c|c|c|c|c|}
\hline \multicolumn{10}{|c|}{$\begin{array}{l}\text { Bases para estabelecer o aumento da } \\
\text { receita }\end{array}$} \\
\hline \multicolumn{2}{|c|}{ Período do orçamento (12 meses) } & 79 & 63 & 98.8 & 91 & 2 & & & \\
\hline \multirow{2}{*}{$\begin{array}{l}\text { Revisão do } \\
\text { orçamento }\end{array}$} & Bimestral & 29 & 48 & & \multirow{2}{*}{89} & 3 & \multirow{2}{*}{70} & \multirow{2}{*}{44} & \multirow{2}{*}{65} \\
\hline & Semestral & 14 & 29 & & & 12 & & & \\
\hline \multirow{2}{*}{\multicolumn{2}{|c|}{$\begin{array}{l}\text { Período do relatório orçamentário } \\
\text { (Mensal) } \\
\text { Itens que fazem parte do pacote orça- } \\
\text { mentário }\end{array}$}} & 77 & 92 & & & & & & \\
\hline & & & & & & & & & \\
\hline \multicolumn{2}{|c|}{ Utiliza orçamento flexível } & 43 & 70 & 15.3 & 16 & & & & \\
\hline \multicolumn{2}{|c|}{ Utiliza orçamento de base zero - OBZ } & 0 & 15 & 49.4 & 52 & & 42 & 7 & \\
\hline \multicolumn{2}{|c|}{ Utiliza Orçamento por atividades - ABB } & 0 & & 14.1 & & & & & \\
\hline \multirow{3}{*}{$\begin{array}{l}\text { Software } \\
\text { (programa) } \\
\text { utilizado pelo } \\
\text { hotel }\end{array}$} & Excel Microsoft & 36 & 78 & & & & & & \\
\hline & Outro programa & 7 & 14 & & & & & & \\
\hline & $\begin{array}{l}\text { Outro programa e } \\
\text { Excel }\end{array}$ & 57 & 6 & & & & & & \\
\hline \multirow{3}{*}{$\begin{array}{l}\text { Utiliza plane- } \\
\text { jamento de } \\
\text { longo prazo }\end{array}$} & Até 3 anos & 61.5 & 22 & & & & & 33.3 & \\
\hline & De 3 a 5 anos & 0 & 27 & & & & & & \\
\hline & Mais de 5 anos & 7 & 13 & & & & 76 & 29.6 & 33.3 \\
\hline
\end{tabular}

Fonte: Dados da revisão.

O segundo estudo, de Winata e Mia (2005), foi realizado em 74 hotéis da Austrália sobre o papel de gerentes no uso da tecnologia da informação e comunicação em relação ao desempenho percebido e sua participação no orçamento. Os resultados indicam que o desempenho dos gerentes de departamentos é percebida como positiva quando associada com a interação do uso da TIC e a participação no orçamento.

O terceiro trabalho realizado por Yuen (2006) pesquisou nos hotéis de Macau na China os efeitos relacionados à satisfação e à participação dos empregados da gestão participativa no processo orçamentário. Entre os resultados encontrados no estudo está a constatação de que a maior participação dos empregados aumenta a responsabilidade e as experiências.

Conforme as principais práticas pesquisadas, evidencia-se que são limitados os estudos sobre métodos de orçamento em países em desenvolvimento como o Brasil, o que também justifica a presente pesquisa.

\section{METODOLOGIA}

Nesta seção, apresentam-se os procedimentos metodológicos utilizados para a construção do referencial teórico e o processo de seleção das empresas hoteleiras.

\section{PROCESSO PARA CONSTRUÇÃO DO REFERENCIAL TEÓRICO}

O processo utilizado para seleção e análise de referências bibliográficas internacionais está dividido em três fases: escolha das bases de dados, seleção de artigos e análise sistêmica (AFONSO et al., 2012; AZEVEDO et al., 2011; BORTOLUZZ; ENSSLIN; ENSSLIN; VALMORBIDA, 2011; ENNSLIN et al., 2010; LACERDA; ENSSLIN; ENSSLIN, 2011; LACERDA; ENSSLIN; ENSSLIN, 2012; ROSA; ENSSLIN; ENSSLIN, 2009; TASCA et al., 2010). A primeira fase fundamenta a escolha das bases de dados; a segunda obtém um portfólio de artigos a partir de processo estruturado e fundamentado e, por fim, tem-se a terceira fase para proporcionar a análise sistêmica do portfólio de artigos.

Primeiramente, foi realizada uma consulta nos periódicos da CAPES, sendo pesquisadas as seguintes coleções: OneFile (GALE) (278), SciVerse ScienceDirect (Elsevier) (6), Social Sciences Citation Index (Web of Science) (4), Emerald Management e Journals (2), e MEDLINE (NLM) (1), utilizando as 
palavras chave "budgeting" e "hotel". Foram selecionados 284 artigos. A partir desta amostra inicial foram lidos todos os resumos, visando selecionar os artigos relacionados às práticas de orçamento em empresas hoteleiras. Os artigos selecionados formam a base do referencial teórico.

Para a revisão nacional foram selecionadas revistas presentes no Qualis da CAPES que contivessem as palavras "Contábil", "Administração", "Negócios ou Business", "Finanças" e "Gestão". Nas revistas selecionas efetuou-se a busca de artigos com a palavra-chave "orçamento". Na revisão nacional não foram encontrados artigos alinhados. Não houve a delimitação de tempo, ou seja, os artigos buscados nas bases poderiam ser de qualquer ano.

\section{PROCEDIMENTO PARA ELABORAÇÃO DO QUESTIONÁRIO}

O questionário foi elaborado a partir das principais características apontadas na revisão de literatura (SCHMIDGALL; NINEMEIER, 1986, 1987 e 1989; SCHMIDGALL et al., 1996; SCHMIDGALL; DeFRANCO, 1998; JONES, 1998, 2008a e 2008b; SUBRAMANIAM et al., 2002; WINATA; MIA, 2005; YUEN, 2006 e 2007; PAVLATOS; PAGGIOS, 2009; STEED; GU, 2009; UYAR; BILGIN, 2011 ; CASTRO el al., 2012). Estas principais características apontadas na literatura estão ilustradas no Quadros 2.

Quadro 2 - Principais características no orçamento

\begin{tabular}{|c|c|}
\hline \multicolumn{2}{|c|}{ Principais Características do Orçamento } \\
\hline Manual de Orçamento & $\begin{array}{l}\text { Inclui diretrizes, objetivos, metas e indicadores previamente definidos pela alta } \\
\text { administração. }\end{array}$ \\
\hline $\begin{array}{l}\text { Comitê de Orça- } \\
\text { mento }\end{array}$ & $\begin{array}{l}\text { Pode incluir os proprietários, diretores e gerentes gerais, chefes de departamen- } \\
\text { tos, controller, etc. }\end{array}$ \\
\hline \multirow{3}{*}{$\begin{array}{l}\text { Formas de elabo- } \\
\text { ração }\end{array}$} & $\begin{array}{l}\text { Top-down - o orçamento é elaborado pela alta administração e imposto aos } \\
\text { demais membros da organização. }\end{array}$ \\
\hline & $\begin{array}{l}\text { Bottom-up - o orçamento é elaboração pelos centros ou departamentos respon- } \\
\text { sáveis e encaminhado à alta administração para a aprovação. }\end{array}$ \\
\hline & $\begin{array}{l}\text { Combinação - o orçamento é elaborado por meio de um processo interativo entre } \\
\text { centros responsáveis e alta administração. }\end{array}$ \\
\hline Período & Menos de 1 ano, 1 ano, plurianual. \\
\hline Revisão & Mensal, trimestral e semestral, etc. \\
\hline \multirow{6}{*}{ Tipo de Orçamento } & Orçamento Público \\
\hline & Orçamento Empresarial \\
\hline & Orçamento de Base Zero \\
\hline & Orçamento Flexível \\
\hline & Orçamento por atividades \\
\hline & Beyond budgeting \\
\hline \multirow{5}{*}{$\begin{array}{l}\text { Objetivos Preten- } \\
\text { didos }\end{array}$} & Previsões de futuro \\
\hline & \begin{tabular}{|l|} 
Maximizar resultados \\
\end{tabular} \\
\hline & Introduzir novos produtos e serviços \\
\hline & Planificar, controlar e valorar o resultado \\
\hline & Criar incentivos e recompensas \\
\hline \multirow{7}{*}{ Finalidades } & Auxiliar no planejamento de longo prazo \\
\hline & Auxiliar no planejamento de curto prazo \\
\hline & Avaliar o desempenho \\
\hline & Coordenar as operações \\
\hline & Motivar os funcionários \\
\hline & Comunicar os planos \\
\hline & Controle \\
\hline \multirow{5}{*}{$\begin{array}{l}\text { Informações utiliza- } \\
\text { das na elaboração }\end{array}$} & Resultados dos anos anteriores \\
\hline & Estatística \\
\hline & Análise de mercado \\
\hline & Indicadores econômicos nacionais \\
\hline & Indicadores econômicos locais e regionais \\
\hline
\end{tabular}

Fonte: Elaborado pelos autores com base na revisão. 
A partir das características do Quadro 2 foram elaboradas as questões de pesquisa. A aplicação do questionário foi realizada em duas etapas: a primeira envolveu a caracterização dos hotéis situados na cidade de Balneário Camboriú, incluindo 9 questões; a segunda envolveu 15 questões acerca do planejamento do orçamento, 4 tópicos referentes à execução do orçamento, com a utilização da escala Likert, e 7 questões objetivas sobre a avaliação e o controle de desempenho organizacional.

\section{POPULAÇÃO E AMOSTRA}

A população inicial é constituída pelas empresas hoteleiras associadas à ASSOCIAÇÃO DA INDÚSTRIA HOTELEIRA DE SANTA CATARINA (ABIH-SC), totalizando 17 instituições. A identificação dos hotéis que preparam orçamento foi realizada por meio de contato telefônico. Caso a resposta fosse negativa, solicitavam-se informações a respeito da instituição, visando identificar na pesquisa as características dos hotéis de Balneário Camboriú que não aplicam o processo orçamentário em sua gestão.

A partir das respostas, foram identificadas 11 empresas hoteleiras que aplicam o orçamento, sendo que para estas empresas hoteleiras foi enviado por e-mail, ao responsável pelo processo, 0 questionário solicitando as informações sobre as práticas orçamentárias adotadas, com intuito de obter as características dos hotéis na cidade de Balneário Camboriú (Santa Catarina - Brasil). Nesta segunda etapa, obtiverem-se 6 respostas advindas destas empresas, totalizando 54,5\% da amostra.

\section{RESULTADOS DO ESTUDO}

Para a discussão sob o prisma das práticas de orçamento no Brasil, primeiramente será feita apresentação do município de Balneário Camboriú, seguida da caracterização dos hotéis, do planejamento, da execução e do controle orçamentário.

\section{APRESENTAÇÃO DO MUNICÍPIO DE BALNEÁRIO CAMBORIÚ}

Balneário Camboriú se destaca por possuir belas praias e contar com ampla estrutura de boa culinária e bares com música ao vivo por toda orla. Além disso, a cidade possui atrativos turísticos modernos e inovadores e um comércio atuante durante o ano inteiro. Por suas belezas naturais, data-se 1926 o início de seu desenvolvimento, com o surgimento das primeiras casas de veraneio e dois anos depois o primeiro empreendimento hoteleiro (SECRETARIA DE TURISMO DE BALNEÁRIO CAMBORIÚ, 2013).

Segundo o IBOPE (INSTITUTO BRASILEIRO DE OPINIÃO PÚBLICA E ESTATÍSTICA), em 2011 o Estado de Santa Catarina foi eleito, pela quinta vez, o melhor destino turístico do Brasil. Balneário Camboriú é conhecida como a Capital Catarinense do Turismo. Localizada no litoral norte do Estado, conta com aproximadamente $46,489 \mathrm{~km}^{2}$ de área e população de 108.107 habitantes. Possui um intenso movimento turístico durante todo o verão, recebendo no ano de 2011 uma população flutuante de 4.453.730 de turistas, principalmente argentinos, gaúchos e paulistas (PREFEITURA MUNICIPAL DE BALNEÁRIO CAMBORIÚ, 2012).

Por possuir o turismo como principal atividade econômica, o município oferece vários atrativos, em que se destacam pontos turísticos como o Cristo Luz, passeios de Escuna e o Parque Unipraias, único no mundo que liga duas praias por meio de bondinhos. Além disso, a cidade oferece também diversidade de estilos em eventos noturnos, ampla rede hoteleira e gastronomia variada (SECRETARIA DE TURISMO DE BALNEÁRIO CAMBORIÚ, 2013).

\section{CARACTERIZAÇÃO DOS HOTÉIS}

Para caracterizar os hotéis da cidade de Balneário Camboriú, foram feitas questões acerca da categoria da organização. Além disso, foi questionada a denominação utilizada pelos hotéis.

Por meio da Tabela 1, percebe-se a denominação utilizada por cada organização, percebendo-se que $82 \%$ dos respondentes utilizam a nomenclatura hotel. A pousada foi a segunda denominação mais utilizada, representando $13 \%$ da amostra. 
Observa-se que a maioria das organizações pesquisadas são constituídas sob a forma de empresa privada, compreendendo um percentual de $65 \%$ do total. Os hotéis que pertencem a uma rede formam $35 \%$ do total.

Verifica-se que os hotéis, em sua maioria, apresentam uma capacidade de hospedagem de 100 quartos, $47 \%$ deles possuem entre 100 e 300 quartos.

Tabela 1 - Informações acerca dos hotéis de Balneário Camboriú

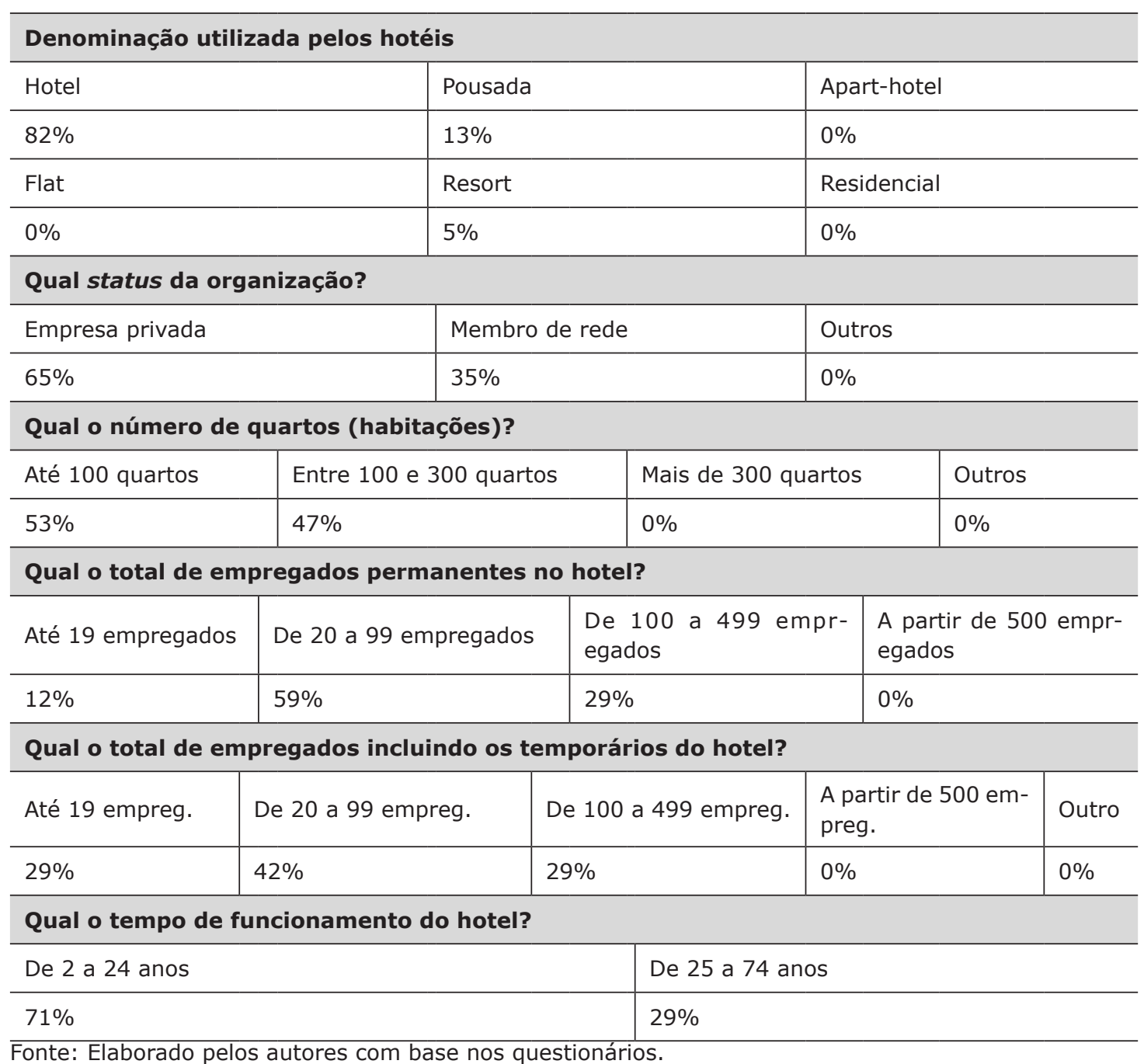

Contudo essas informações refletem na quantidade de empregados constantes em cada organização, com ou sem temporários. Sendo assim, constata-se que, devido à predominância de pequeno número de quartos, a maioria dos hotéis possui entre 20 a 99 empregados. Ademais, percebe-se que $71 \%$ dos hotéis selecionados possuem entre 2 e 24 anos de experiência no ramo hoteleiro, enquanto que apenas $29 \%$ afirmam ter aberto para funcionamento a mais de 25 anos.

A Tabela 2 permite visualizar a taxa média de ocupação dos hotéis de Balneário Camboriú no intervalo de $20 \%$ a $95 \%$ e a quantidade de leitos aproximada de cada unidade.

Tabela 2 - Taxa média de ocupação e quantidade de leitos

\begin{tabular}{l|l|l}
\hline \multicolumn{2}{|c}{ Taxa média de ocupação dos hotéis da amostra } & $55 \%$ a $60 \%$ \\
\hline $80 \%$ a $95 \%$ & $65 \%$ a $75 \%$ & 3 \\
\hline 0 & 1 & $20 \%$ a $30 \%$ \\
\hline $45 \%$ a $50 \%$ & $35 \%$ a $40 \%$ & 2 \\
\hline 6 & 5 & \\
\hline
\end{tabular}




\begin{tabular}{l|l|l}
\hline \multicolumn{2}{l}{ Quantidade de leitos nos hotéis } \\
\hline 12 a 40 leitos & 40 a 80 leitos & 80 a 150 leitos \\
\hline 2 & 1 & 5 \\
\hline 150 a 300 leitos & 300 a 900 leitos & Acima de 2680 leitos \\
\hline 6 & 3 & 0 \\
\hline
\end{tabular}

Fonte: Elaborado pelos autores com base nos questionários.

As taxas médias de ocupação foram preenchidas por no mínimo 5 hotéis, permitindo constatar que existe uma grande variação, desde $20 \%$ até $75 \%$. Há predominância de 6 hotéis no intervalo de taxa média de ocupação entre $45 \%$ a $50 \%$, seguido do intervalo de $35 \%$ a $40 \%$ com 5 hotéis. Apenas 2 hotéis afirmaram ter ocupação entre $20 \%$ e $30 \%$.

A última questão da primeira etapa do questionário aplicado indagava se o hotel possuía um processo formal de orçamento, com opções de resposta "sim" e "não". Percebeu-se que mais de $60 \%$ utilizam o orçamento. Por outro lado, há hotéis que afirmaram não usar um processo formal de orçamento, representando $35 \%$ da população inicial. Isto corrobora com Schmidgall e DeFranco (1998) e Steed e Gu (2009), que realizaram estudos nos hotéis dos Estados Unidos; assim como Pavlatos e Paggios (2009), que realizaram na Grécia, percebendo que o orçamento é amplamente utilizado pelas empresas.

\section{PLANEJAMENTO DO ORÇAMENTO}

Os resultados a partir deste item estão relacionados às empresas hoteleiras que aplicam o processo orçamentário. Na Tabela 3, apresenta-se um rol de questões aplicadas aos hotéis relacionados ao planejamento orçamentário.

Tabela 3 - Características do processo orçamentário

\begin{tabular}{l|l}
\hline \multicolumn{2}{l}{ O hotel utiliza "manual de orçamento" para a elaboração do processo orçamentário? } \\
\hline Sim & Não \\
\hline $33 \%$ & $67 \%$ \\
\hline O hotel possui Comitê de orçamento? & Não \\
\hline Sim & $67 \%$ \\
\hline $33 \%$ &
\end{tabular}

Quantas horas são necessárias para preparar e revisar o orçamento?

\begin{tabular}{l|l|l|l|l}
\hline Menos de 40 horas & De 41 a 80 horas & \multicolumn{1}{l}{ De 81 a 120 horas } & \multicolumn{2}{l}{ Mais de 120 horas } \\
\hline $33 \%$ & $67 \%$ & - & - \\
\hline $\begin{array}{l}\text { Como o processo orçamentário é elaborado? } \\
\text { Bottom-up (elaborados pelos centros } \\
\text { operacionais) }\end{array}$ & $\begin{array}{l}\text { Top-down (elaborado pela } \\
\text { alta administração) }\end{array}$ & $\begin{array}{l}\text { Combinação/Participativa } \\
\text { (elaboração conjunta) }\end{array}$ & Outros \\
\hline $66 \%$ & $17 \%$ & $17 \%$ & - \\
\hline Para qual horizonte de tempo é elaborado o orçamento? & 6 meses & 1 ano & Outros \\
\hline 1 mês & 3 meses & $17 \%$ & - & $83 \%$ \\
\hline-
\end{tabular}




\begin{tabular}{|c|c|c|c|c|c|c|c|c|c|}
\hline \multicolumn{2}{|l|}{$0 \%$} & \multicolumn{3}{|l|}{$50 \%$} & \multicolumn{3}{|c|}{ - } & \multicolumn{2}{|c|}{$50 \%$} \\
\hline \multicolumn{10}{|c|}{ Qual a periodicidade dos relatórios orçamentários? } \\
\hline \multicolumn{2}{|l|}{ Mensal } & \multicolumn{3}{|l|}{ Semestral } & \multicolumn{3}{|c|}{ Anual } & \multicolumn{2}{|c|}{ Outros } \\
\hline \multicolumn{2}{|l|}{$83 \%$} & \multicolumn{3}{|l|}{-} & \multicolumn{3}{|l|}{-} & \multicolumn{2}{|c|}{$17 \%$} \\
\hline \multicolumn{10}{|c|}{ O hotel faz alterações decorrentes do volume - orçamento flexível? } \\
\hline \multicolumn{5}{|l|}{ Sim } & \multicolumn{5}{|l|}{ Não } \\
\hline \multicolumn{5}{|l|}{$34 \%$} & \multicolumn{5}{|c|}{$66 \%$} \\
\hline \multicolumn{10}{|c|}{$\begin{array}{l}\text { Qual o programa (software) ou tecnologia utilizada pelo hotel na elaboração do processo } \\
\text { orçamentário? }\end{array}$} \\
\hline Excel Microsoft & \multicolumn{3}{|c|}{ Internet corporativa } & \multicolumn{5}{|c|}{ Excel Microsoft e outro programa } & Outros \\
\hline $67 \%$ & \multicolumn{3}{|c|}{-} & \multicolumn{5}{|c|}{$33 \%$} & - \\
\hline \multicolumn{10}{|c|}{ O hotel elabora e pratica o planejamento estratégico? } \\
\hline \multicolumn{3}{|l|}{ Elabora e aplica } & \multicolumn{4}{|c|}{ Elabora mas não aplica } & \multicolumn{3}{|c|}{ Não elabora } \\
\hline \multicolumn{3}{|l|}{$17 \%$} & \multicolumn{2}{|c|}{$17 \%$} & & & \multicolumn{3}{|l|}{$66 \%$} \\
\hline \multicolumn{10}{|c|}{ Se sim, qual o horizonte de planejamento estratégico? } \\
\hline 1 ano & \multicolumn{3}{|c|}{3 anos } & \multicolumn{2}{|c|}{5 anos } & \multicolumn{3}{|c|}{ Mais de 5 anos } & Outros \\
\hline $50 \%$ & - & & & - & & 50 & & & $-\%$ \\
\hline Qual método ou tip & oo de or & amento o & hot & el utili & & & & & \\
\hline $\begin{array}{l}\text { Orçamento empre- } \\
\text { sarial }\end{array}$ & Orçam & ento flexíve & & $\begin{array}{l}\text { Orçar } \\
\text { contín }\end{array}$ & ento & & nto b & ase & Outros \\
\hline $83 \%$ & $17 \%$ & & & - & & - & & & - \\
\hline
\end{tabular}

Fonte: Elaborado pelos autores com base nos questionários.

Percebe-se que não é comum a prática de criar um Comitê de orçamento, apenas 33\% dos respondentes possuem o referido Comitê. Diferentemente disso, Uyar e Bilgin (2011) concluíram que na Região de Antalya, na Turquia, a comissão e o manual de orçamento são comuns em hotéis turcos. Steed e Gu (2009) observaram que nos Estados Unidos o orçamento era elaborado em planilha eletrônica, e com disposição dos dados mensal.

Constata-se que o tempo gasto para elaboração do orçamento geralmente é curto, envolvendo menos de 40 horas em 33\% dos hotéis e $67 \%$ entre 41 e 80 horas.

O processo orçamentário em empresas hoteleiras do Brasil é elaborado de forma predominantemente bottom-up, com 66\% e a combinação/participativa $17 \%$ dos casos. Estes resultados corroboram com os de Schmidgall e Ninemeier (1987) em empresas hoteleiras dos Estados Unidos, onde a prática bottom-up também é utilizada pela grande maioria. Schmidgall et al. (1996) compararam as práticas orçamentárias dos hotéis dos Estados Unidos e da Escandinávia, concluindo que as empresas utilizavam basicamente abordagens bottom-up. Diferentemente disso, ao realizar pesquisas nos hotéis do Reino Unido, Jones (2008) chegou à conclusão de que nenhuma das abordagens (bottom-up, top-down e combinação/participativa) foi predominante. Uyar e Bilgin (2011) observaram que na Turquia é diferente, assim como Steed e Gu (2009) obtiveram a mesma conclusão nos Estados Unidos, de que a maioria dos hotéis utiliza a abordagem combinação/participativa.

Em geral, o orçamento é do tipo empresarial e elaborado para o período de um ano, sendo que apenas $17 \%$ asseguraram utilizar prazos diferentes. Assim como Schmidgall e DeFranco (1998), nos Estados Unidos; Jones (1998), no Reino Unido; Uyar e Bilgin (2011) na Turquia, também concluíram que comumente os hotéis elaboram o orçamento para o prazo de um ano.

No que diz respeito à aplicação dos tipos de orçamento, os resultados mostram baixa utilização de métodos como orçamento de base zero e orçamento flexível. Se comparar aos estudos de Jones (1998 e 2008) realizados em hotéis do Reino Unido, constatou que o orçamento flexível era pouco 
utilizado, enquanto que o orçamento de base zero é aplicado em metade das empresas pesquisadas, caindo um pouco no segundo estudo. Da mesma forma, Pavlatos e Paggios (2009) realizaram estudos na Grécia, e verificaram que a utilização do orçamento de base zero era significativa, enquanto a aplicação do orçamento flexível e por atividades era bastante baixa.

Quanto à revisão, obtiveram-se os resultados, $50 \%$ trimestralmente, $50 \%$ outros. Ademais, $57 \%$ dos respondentes afirmaram não fazer alterações decorrentes do volume, enquanto $66 \%$ fazem ajustes em decorrência de variações nos níveis de ocupação. Segundo Uyar e Bilgin (2011), na Turquia; e Jones (1998 e 2008) no Reino Unido, observaram que as revisões são predominantemente trimestrais e na maioria dos casos em base mensal ou trimestral.

\section{EXECUÇÃO DO ORÇAMENTO}

Entre os resultados alcançados na parte de execução do orçamento, 17\% destacam o bom fluxo de informações, além de $83 \%$ dos respondentes concordarem parcialmente com a questão, conforme apresenta a Tabela 4.

Tabela 4: Resultados da execução do orçamento

\begin{tabular}{l|l|l|l|l|l}
\hline EXECUÇÃo DO ORÇAMENTO & $\begin{array}{c}\text { Discordo } \\
\text { Totalmente }\end{array}$ & $\begin{array}{c}\text { Discordo } \\
\text { Parcialmente }\end{array}$ & $\begin{array}{l}\text { Indiferente } \\
\text { Parcialmente }\end{array}$ & $\begin{array}{c}\text { Concordo } \\
\text { Plenamente }\end{array}$ \\
\hline $\begin{array}{l}\text { Orçamento é considerado um } \\
\text { segredo da alta direção }\end{array}$ & $17 \%$ & $17 \%$ & $0 \%$ & $17 \%$ & $50 \%$ \\
\hline $\begin{array}{l}\text { Uma liderança eficaz é for- } \\
\text { necida no processo orçamen- } \\
\text { tário }\end{array}$ & $0 \%$ & $17 \%$ & $0 \%$ & $0 \%$ & $83 \%$ \\
\hline $\begin{array}{l}\text { Bom fluxo de informações } \\
\text { está disponível para o orça- } \\
\text { mento }\end{array}$ & $0 \%$ & $0 \%$ & $0 \%$ & $83 \%$ & $17 \%$ \\
\hline $\begin{array}{l}\text { Coordenação entre os de- } \\
\text { partamentos é conseguida } \\
\text { facilmente }\end{array}$ & $0 \%$ & $0 \%$ & $0 \%$ & $17 \%$ & $83 \%$ \\
\hline $\begin{array}{l}\text { Metas são determinadas } \\
\text { primeiro nos departamentos } \\
\text { e depois submetidas à alta } \\
\text { direção }\end{array}$ & $83 \%$ & $0 \%$ & $0 \%$ & $17 \%$ & $0 \%$ \\
\hline $\begin{array}{l}\text { Metas são determinadas pela } \\
\text { alta direção e depois são } \\
\text { coletadas opiniões nos depar- } \\
\text { tamentos }\end{array}$ & $0 \%$ & $83 \%$ & $0 \%$ & $17 \%$ & $06 \%$ \\
\hline $\begin{array}{l}\text { Metas são fruto de um proces- } \\
\text { so estruturado e alinhado com } \\
\text { o Planejamento Estratégico }\end{array}$ & $0 \%$ & $0 \%$ & $34 \%$ & & $0 \%$ \\
\hline $\begin{array}{l}\text { Fonte: Elaborado pelos autores } \\
\text { Plom }\end{array}$ & & & & & \\
\hline
\end{tabular}

Fonte: Elaborado pelos autores com base nos questionários.

Na parte dos objetivos pretendidos com o orçamento, observa-se uma grande homogeneidade entre os entrevistados, revelando o controle como uma das principais finalidades, do mesmo modo que revelou as pesquisas feitas por Schmidgall et al. (1996) e Schmidgall e DeFranco (1998) nas empresas hoteleiras dos Estados Unidos e Escandinávia. 
Os resultados apontam, conforme Tabela 5, que nenhum dos hotéis pesquisados utiliza-se das ferramentas propostas pelo Benchmarking na avaliação do orçamento. A utilização concentra-se $66 \%$ em pontos de referencias que, em sua maioria, utiliza o valor monetário. Assim, como apresentam as considerações das pesquisas de Jones (1998 e 2008) realizadas em empresas hoteleiras no Reino Unido, os resultados alcançados no presente estudo revelam que o papel do orçamento no monitoramento do desempenho real da organização é o de principal indicador de desempenho.

Tabela 5 - Avaliação do orçamento

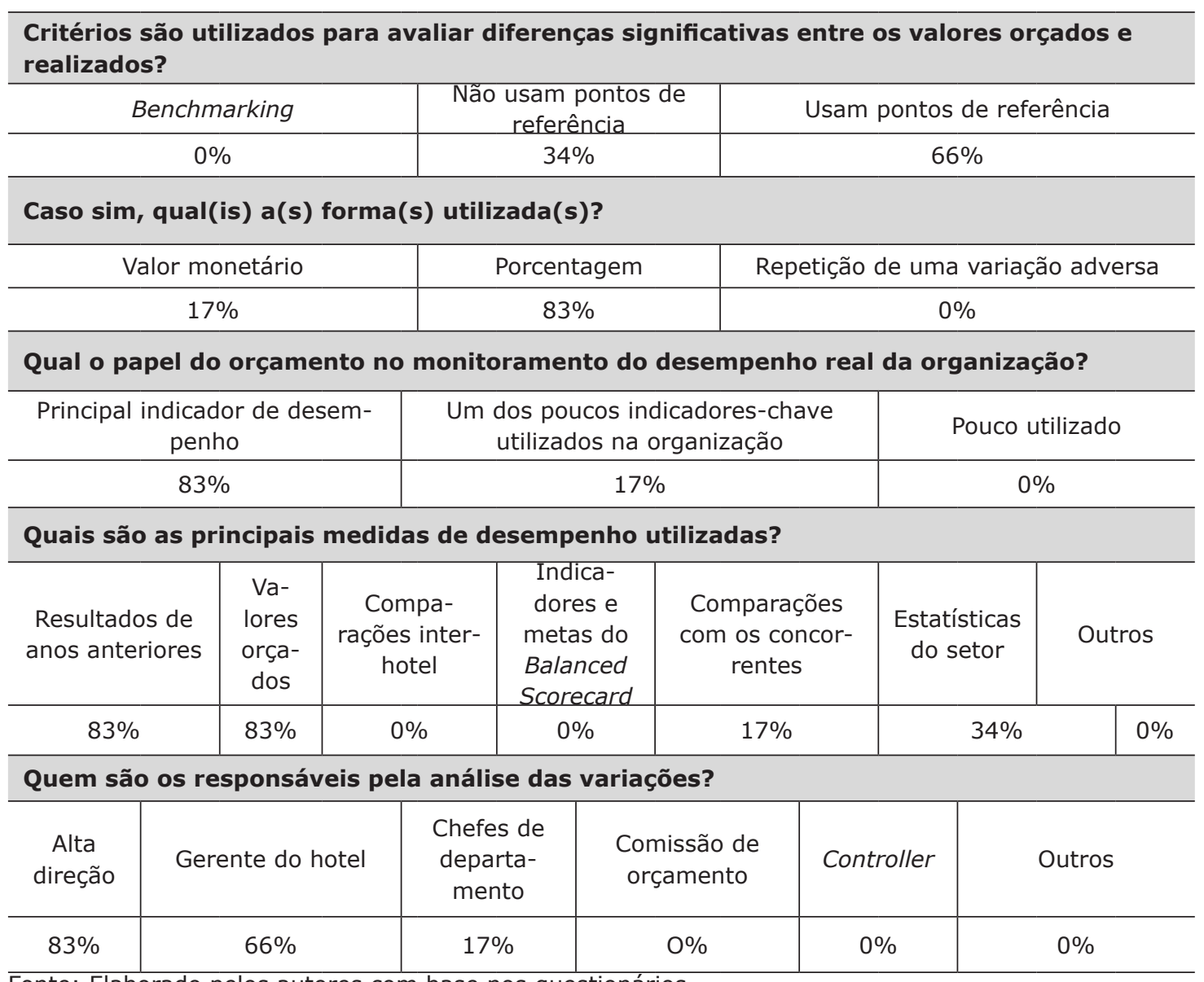

Fonte: Elaborado pelos autores com base nos questionários.

Entre as principais medidas de desempenho utilizadas estão: resultados de anos anteriores com $83 \%$, valores orçados com $83 \%$ e ainda estatísticas do setor $34 \%$. Entre as opções de respostas a do Balanced Scorecard não recebeu nenhum indicação, o que mostra, possivelmente, uma falta de conhecimento e não utilização por parte dos gestores desta ferramenta de avaliação de desempenho.

Conforme observado em estudos anteriores como o de Schmidgall et al. (1996), uma das principais práticas em relação à adoção de um orçamento é a análise das variações, o que trará à empresa a possibilidade de aplicar ações corretivas sobre problemas detectados.

A análise das variações é feita mensalmente em $83 \%$ dos casos, como apresenta a Tabela 6. Suas finalidades principais estão voltadas para o controle dos custos/despesas, assim como revelou a pesquisa de Schmidgall et al. (1996) em sua comparação entre as empresas hoteleiras dos Estados Unidos e Escandinávia. Outros pontos destacados foram a identificação de um problema potencial a tempo de evitá-lo e fornecer feedback para o orçamento do próximo período, sendo os percentuais de concordância, $66 \%, 83 \%$ e $83 \%$, respectivamente. 
Tabela 6 - Análise das variações

\begin{tabular}{|c|c|c|c|c|c|c|c|c|}
\hline \multicolumn{9}{|c|}{ A análise da variação acontece: } \\
\hline Mensalmente & \multicolumn{2}{|c|}{ Trimestralmente } & \multicolumn{2}{|c|}{ Semestralmente } & \multicolumn{2}{|c|}{ Anualmente } & \multicolumn{2}{|c|}{ Outros } \\
\hline $83 \%$ & \multicolumn{2}{|l|}{$17 \%$} & \multicolumn{2}{|l|}{$0 \%$} & \multicolumn{2}{|c|}{$0 \%$} & \multicolumn{2}{|l|}{$0 \%$} \\
\hline \multicolumn{9}{|c|}{ Quais as finalidades da análise das variações do orçamento? } \\
\hline $\begin{array}{l}\text { Avaliar o } \\
\text { desempenho } \\
\text { do gerente }\end{array}$ & $\begin{array}{l}\text { Avaliar a ca- } \\
\text { pacidade de } \\
\text { previsão do } \\
\text { gerente }\end{array}$ & \multicolumn{2}{|c|}{$\begin{array}{l}\text { Controlar } \\
\text { os custos/ } \\
\text { despesas }\end{array}$} & \multicolumn{2}{|c|}{$\begin{array}{l}\text { Identificar um prob- } \\
\text { lema potencial a tem- } \\
\text { po de evitá-lo }\end{array}$} & \multicolumn{2}{|c|}{$\begin{array}{l}\text { Fornecer feedback } \\
\text { para o orçamento } \\
\text { do próximo perío- } \\
\text { do }\end{array}$} & Outros \\
\hline $0 \% \%$ & $0 \%$ & \multicolumn{2}{|c|}{$66 \%$} & \multicolumn{2}{|l|}{$83 \%$} & \multicolumn{2}{|c|}{$83 \%$} & $0 \%$ \\
\hline
\end{tabular}

Fonte: Elaborado pelos autores com base nos questionários.

Assim como nos estudos empíricos realizados anteriormente em outros países, nos hotéis da presente pesquisa o orçamento é abundantemente utilizado como um dos instrumentos mais importantes para os gestores no que tange ao planejamento e ao controle nas organizações hoteleiras (ABDEL-KADER; LUTHER, 2006; UYAR, 2009; SIVABALAN et al., 2009; LIBBY; LINDSAY, 2010; OSTERGREN; STENSAKER, 2011; UYAR; BILGIN, 2011).

\section{DISCUSSÕES E CONCLUSÕES}

Conclui-se que o objetivo deste artigo foi alcançado, considerando que a aplicação dos questionários permitiu explorar as práticas orçamentárias em empresas hoteleiras da Cidade de Balneário Camboriú-SC (Brasil). Foi possível caracterizar os referidos hotéis, bem como observar o planejamento, a execução e o controle do orçamento.

Observou-se que a maioria dos hotéis não possui classificação definida e utiliza a denominação hotel. A pousada foi a segunda nomenclatura mais utilizada, representando $12 \%$. Verificase que a maioria dos hotéis possui menos de 100 quartos por unidade e até 99 empregados. Predominantemente, os respondentes informaram que os hotéis possuem entre 100 e 300 leitos em uma unidade.

Ademais, $71 \%$ dos hotéis selecionados foram fundados entre 2 e 24 anos, de tal modo que mais de $64 \%$ utiliza o orçamento. Desses $64 \%, 67 \%$ somente utilizam o Excel Microsoft e $33 \%$ utilizam outra ferramenta além desta. Semelhante a Castro et al. (2012) em hotéis de Florianópolis, observou-se que o tempo gasto para elaboração do orçamento é relativamente curto. Tais resultados contrastam com os de Uyar e Bilgin (2011) em uma região turisticamente desenvolvida na Turquia, em que $80 \%$ dos pesquisados afirmam usar mais de 120 horas para a elaboração do orçamento.

O processo orçamentário mais utilizado é o bottom-up, o que corrobora com os estudos de Schmidgall e Ninemeier (1987) nos Estados Unidos; e Schmidgall et al. (1996) nos Estados Unidos e na Escandinávia. Já no Reino Unido, Jones (2008) chegou à conclusão de que nenhuma das abordagens (bottom-up, top-down e combinação/participativa) foi predominante. Uyar e Bilgin (2011) observaram que na Turquia, assim como Steed e Gu (2009) nos Estados Unidos, e concluíram que a maioria dos hotéis utiliza a abordagem combinação/participativa. As pesquisas anteriores apresentavam indícios de que a forma de elaboração do orçamento estava mudando com o tempo, para uma maior participação dos colaborados no processo, embora esta pesquisa realizada no Brasil confirma que ainda há uma grande centralização do processo orçamentário na alta direção do hotel. A condução pelo processo bottom-up contribui para a utilização de informações geralmente disponíveis em níveis operacionais.

Quanto às informações utilizadas na elaboração do orçamento, pode-se observar que as informações estatísticas apresentaram uma menor utilização nos hotéis da amostra. Deste modo, evidencia-se que os orçamentos são baseados nos indicadores econômicos e nos resultados dos anos anteriores. Contudo Steed e Gu (2009) perceberam que nos hotéis dos Estados Unidos as diretrizes estão baseadas também no REVPAR (aumento percentual), além de estarem baseadas no percentual de aumento em relação ao ano anterior. 
Constatou-se que a motivação dos gestores dos hotéis está muito atrelada a sua participação na elaboração do orçamento e no alcance dos objetivos planejados. Nesta pesquisa, os responsáveis pela análise das variações concentram-se entre os chefes de departamento, com análises mensais voltadas ao controle dos custos/despesas, de forma a verificar se o orçamento foi aplicado eficientemente conforme o planejado. Tal finalidade na análise de variações concorda com a pesquisa de Schmidgall et al. (1996).

De forma geral, os resultados mostram que o orçamento em empresas hoteleiras no Brasil ainda é subutilizado, com aplicação parcial de muitas de suas características e técnicas. Ao analisar os resultados da presente pesquisa, pode-se observar a ausência de algumas das práticas destacadas por Umapathy (1987), que segundo o autor são fundamentais na aplicação do processo orçamentário e adotadas por empresas de sucesso. Adicionalmente, destaca-se a importância da conjunção de subsistemas como o de planejamento, controle e contábil para o alcance dos objetivos estratégicos, ou seja, é mais do que a utilização de práticas sofisticadas de orçamento (TELES, 2011).

Apesar de o orçamento flexível ser o recomendado para ser utilizado nesse segmento do mercado, a pesquisa apontou que $50 \%$ dos hotéis utilizam o orçamento empresarial. Existem discussões sobre esse assunto, mas o orçamento empresarial continua ainda a ser amplamente utilizado nas organizações, como visto nesse estudo e nos outros similares (JONES, 2008; UYAR; BILGIN, 2011).

Para futuras pesquisas, sugere-se que sejam observadas as práticas orçamentárias de outras regiões do país. Adicionalmente, recomendam-se analisar questões acerca do orçamento de capital e do desempenho ambiental das empresas hoteleiras.

\section{REFERÊNCIAS}

ABDEL-KADER, M.; LUTHER, R. Management accounting practices in the British drink and food industry, British Food Journal 108 (5): 336-57, 2006.

ADAMS, D. Management Accounting for the Hospitality, Tourism and Leisure Industries: A Strategic Approach. 2nd ed. London: Thomson Learning, 2006.

AFONSO, M. H. F.; SOUZA, J. V.; ENSSLIN, S. R.; ENSSLIN, L. Como construir conhecimento sobre o tema de pesquisa? Aplicação do processo Proknow- $C$ na busca de literatura sobre avaliação do desenvolvimento sustentável. RGSA: Revista de Gestão Social e Ambiental, v. 5, pp. 47-62, 2012.

ARAUJO, Aneide Oliveira. Contribuição ao estudo de indicadores de desempenho de empreendimentos hoteleiros, sob o enfoque da gestão estratégica. 2001. Tese (Doutorado em Controladoria e Contabilidade: Contabilidade) - Faculdade de Economia, Administração e Contabilidade, Universidade de São Paulo, São Paulo, 2001. Disponível em: <http://www.teses.usp.br/teses/disponiveis/12/12136/ tde-01032002-115642/>. Acesso em: 2013-04-12.

AZEVEDO, R. C.; ENSSLIN, L.; LACERDA, R. T. O.; FRANÇA, L. A.; GONZALEZ, C. J. I.; JUNGLES, A. E.; ENSSLIN, S. R. Avaliação de desempenho do processo de orçamento: estudo de caso em uma obra de construção civil. Ambiente Construído, v. 11, n. 1, pp. 85-104, 2011.

BORTOLUZZI, S. C.; ENSSLIN, S. R.; ENSSLIN, L.; VALMORBIDA, S. M. I. Avaliação de desempenho em redes de pequenas e médias empresas: estado da arte para as delimitações postas pelo pesquisador. $\mathbf{R}$. Eletr. Estrat. Neg., v. 4, n. 2, pp. 202-222, 2011.

CASTRO, J.K.; SANTOS, G.R.; SOUZA, P.; LUNKES, R.J. Práticas Orçamentárias Aplicadas em Empresas Hoteleiras no Brasil: Um Estudo na Cidade de Florianópolis. Revista Estudios y Perspectivas em Turismo. Vol.21 (4), 2012.

DAVILA, A.; FOSTER, G. Management accounting system adoption decisions: evidence and performance implications from earlystage/startup companies. The Accounting Review. 80 (4), 1039-1068, 2005.

DAVILA, A., FOSTER, G. Management control systems in early-stage startup companies. The Accounting Review. 82 (4), 907-937, 2007.

DE WAAL, A.; HERMKENS-JANSSEN, M.; VAN DE VEN, A. The evolutionary adoption framework: Explaining the budgeting paradox. Journal of Accounting and Organizational Change, 7 (4), p. 316-336, 2011. 
EMBRATUR - Instituto Brasileiro de Turismo. Disponível em: http://www.turismo.gov.br/turismo/o_ministerio/embratur/. Acesso em: 15 junho de 2012.

ENSSLIN, L.; GIFFHORN, E., ENSSLIN, S. R.; PETRI, S. M.; VIANNA, W. B. Avaliação do Desempenho de Empresas Terceirizadas com o Uso da Metodologia Multicritério de Apoio à Decisão- Construtivista. Revista Pesquisa Operacional 30(1): 125-152, 2010.

GARRISON, R.H.; NOREEN, E.W.; BREWER, P.C. Managerial Accounting. 12th ed., New York, NY: McGraw-Hill Irwin, 2008.

GOVERNO DO ESTADO DE SANTA CATARINA. Disponível em: http://www.sc.gov.br/conteudo/santacatarina/ roteiros/roteiros/cap11.html. Acesso em: 14 junho 2012.

HANSEN, S. C.; OTLEY, D. T.; VAN DER STEDE, W. A. Practice developments in budgeting: an overview and research perspective. Journal of Management Accounting Research 15, pp. 95-116, 2003.

HARRIS, P. Profit Planning. 2nd ed. Oxford: Butterworth-Heinemann, 1999.

HOPE, J., FRASER, T.; ROSEN, C. Trash the budget. Optimize February(2): 24-31, 2003.

HORNGREN, Charles T.; FOSTER, George; DATAR, Srikant M. Contabilidade de custos. 9. ed. Rio de Janeiro: LTC, 2000.

IBOPE - Instituto Brasileiro de Opinião Pública e Estatística. Notícias. Disponível em: http://www.ibope. com. br/calandraWeb/servlet/CalandraRedirect?temp $=5 \& p r o j=$ PortalIBOPE\&pub $=T \& d b=$ caldb\&comp $=$ No t\%EDcias\&docid=135D9DEF8182784783257930006ABC08. Acesso em 14 de junho de 2012.

JONES, T. A. Improving hotel budgetary practice-A positive theory model. International Journal of Hospitality Management, 27(4): 529-540, 2008a.

JONES, T. A. Changes in hotel industry budgetary practice, International Journal of Contemporary Hospitality Management, 20(4): 428-444, 2008b.

JONES, T. A. UK hotel operators use of budgetary procedures. International Journal of Contemporary Hospitality Management, Vol. 10 (3), p. 96-100, 1998.

KING, R.; CLARKSON, P.M.; WALLACE, S. Budgeting practices and performance in small healthcare businesses. Management Accounting Research, Vol.21 (1), pp. 40-55, 2010.

KORNACKER, J.A.; SCHENTLER, P.B.; WILLIAMS, H.J.C.; MOTWANI, J.C. Critical success factors for budgeting systems in the German context: An empirical analysis. International Journal of Business Excellence, 4 (6), p. 621-644, 2011.

LACERDA, R. T. O.; ENSSLIN, L.; ENSSLIN, S. R. Contribuições à Gestão Estratégica de Organizações quando Analisados na Visão de seu Desempenho. GESTÃO. Org-Revista Eletrônica Gestão Organizacional, v. 2, n. 9, 2011.

LACERDA, R. T. O.; ENSSLIN, L.; ENSSLIN, S. R. Uma Análise Bibliométrica da Literatura Sobre Estratégia e Avaliação De Desempenho. Gestão \& Produção, v. 19, n. 1, 2012.

LIBBY, T.; LINDSAY, R. M. Beyond budgeting or budgeting reconsidered? A survey of North-American budgeting practice. Management Accounting Research, v. 21, p. 56-75, 2010.

OSTERGREN, K.; STENSAKER, I. Management control without budgets: A field study of "Beyond Budgeting" in practice. European Accounting Review, 20(1): 149-181, 2011.

OTLEY, D.T. Management control in contemporary organizations: towards a wider framework. Management Accounting Research. 5, 289-299, 1994.

PAVLATOS, O.; PAGGIOS, I. Management accounting practices in the Greek hospitality industry. Managerial Auditing Journal, 24 (1), p. 81-98, 2009.

PREFEITURA MUNICIPAL DE BALNEÁRIO CAMBORIÚ. A cidade. Disponível em: http://www.balneariocamboriu.sc.gov.br/. Acesso em:14 maio de 2012.

ROSA, F. S.; ENSSLIN, S. R.; ENSSLIN, L. Evidenciação Ambiental: Processo Estruturado de Revisão de Literatura sobre Avaliação de Desempenho da Evidenciação Ambiental. Sociedade, Contabilidade e 
Gestão, v. 4, 2009.

SANDINO, T. Introducing the first management control systems: evidence from the retail sector. The Accounting Review. 82 (1), 265-293, 2007.

SCHMIDGALL, R. S. ; BORCHGREVINK, C. P.; ZAHL-BEGNUM, O. H. Operations budgeting practices of lodging firms in the United States and Scandinavia. International Journal of Hospitality Management, 15 (2), p. 189-203, 1996.

SCHMIDGALL, R.S.; DEFRANCO, A.L. Budgeting and forecasting: Current practice in the lodging industry. Cornell Hotel and Restaurant Administration Quarterly, 39 (6), p. 45-51, 1998.

SCHMIDGALL, R.S., NINEMEIER, J. Budgeting practices in lodging and food service chains: an analysis and comparison. International Journal of Hospitality Management, 8 (1), p. 35-41, 1989.

SCHMIDGALL, R. and NINEMEIER, J. Food-service budgeting: how the chains do it. The Cornell Hotel and Restaurant Administration Quarterly, 26(4): 51-57, 1986.

SCHMIDGALL, R. and NINEMEIER, J. Budgeting in hotel chains: coordination and control, The Cornell Hotel and Restaurant Administration Quarterly, 28(1): 79-84, 1987.

SECRETARIA DE TURISMO DE BALNEÁRIO CAMBORIÚ. Disponível em: http://www.secturbc.com.br/. Acesso em 10/04/13.

SIVABALAN, P., BOOTH, P., MALMI, T; BROWN, D.A. An exploratory study of operational reasons to budget, Accounting and Finance 49(4): 849-871, 2009.

STEED, E.; GU, Z. Hotel management company forecasting and budgeting practices: A survey-based analysis. International Journal of Contemporary Hospitality Management, 21(6): 676-697, 2009.

SUBRAMANIAM, N.; MCMANUS, L.; MIA, L. Enhancing hotel manager's organisational commitment: An investigation of the impact of structure, need for achievement and participative budgeting. International Journal of Hospitality Management, 21 (4), p. 303-320, 2002.

SZYCHTA, A. The scope of application of management accounting methods in Polish enterprises, Management Accounting Research 18, pp. 401-418, 2002.

TASCA, J. E.; ENSSLIN, L.; ENSSLIN, S. R.; ALVES; M. B. M. An approach for selecting a theoretical framework for the evaluation of training programs. Journal of European Industrial Training, v. 34, N. 7, pp. 631-655, 2010.

TELES, João. Práticas da controladoria em hotéis de grande porte do Sul do Brasil. Florianópolis, SC, 2011. 198 p. Dissertação (Mestrado) - Universidade Federal de Santa Catarina, Centro Sócio-Econômico, Programa de Pós-Graduação em Contabilidade, Florianópolis, 2011.

UMAPATHY, S. Current Budgeting Practices in U.S. Industry: the state of the art". Quorum Books, New York, 1987.

UYAR, A. An evaluation of budgeting approaches: traditional budgeting, better budgeting, and beyond budgeting, Journal of Academic Studies 11(42): 113-130, 2009.

UYAR, A.; BILGIN, N. Budgeting practices in the Turkish hospitality industry: An exploratory survey in the Antalya region. International Journal of Hospitality Management, 30 (2), p. 398-408, 2011.

WINATA, L.; MIA, L. Information technology and the performance effect of managers' participation in budgeting: Evidence from the hotel industry. International Journal of Hospitality Management, 24 (1), p. 21-39, 2005.

YUEN, D. Antecedents of budgetary participation: enhancing employees' job performance, Managerial Auditing Journal 22 (5), pp. 533-548, 2007.

YUEN, D. The impact of a budgetary design system: Direct and indirect models. Managerial Auditing Journal, 21 (2), pp. 148-165, 2006. 